\title{
Technical Issues for Realization of Perpendicular Magnetic Recording
}

\author{
Yoshihisa NAKAMURA \\ Research Institute of Electrical Communication, Tohoku University
}

\begin{abstract}
First of all, it is confirmed analytically that perpendicular magnetic recording has a much higher writing resolution which will reach a bit size corresponding to diameters of several magnetic particles when a sharper field gradient of a head and a narrower particle coercive force distribution of a medium are realized. After that, a writing head for perpendicular magnetic recording is discussed from a view point of the sharpness of the head field distribution around the trailing edge and the writing characteristics of a novel single-pole type flying head are presented. Furthermore, several technical issues for practical use of perpendicular magnetic recording such as media noise, thermal relaxation, head induced erasure and so on are discussed.
\end{abstract}

Keywords: perpendicular magnetic recording, single pole head, double-layered medium

\section{INTRODUCTION}

Perpendicular magnetic recording was first proposed, about 20 years ago [1]. These days, many attempts have been made but a recording apparatus utilizing a perpendicularly magnetizing method has still not been practically applied, except for 8-mm VCR and D-VCR, which uses an obliquely oriented metal evaporated (ME) tape. For this reason, it is considered questionable whether perpendicular magnetic recording is the savior for high density recording.

One of many problems which have hesitated to make perpendicular magnetic recording practicable is that a reasonable way to practical use has still not become clear. Especially, guiding principles and clear data have not been presented as to whether heads of a ring type or a singlepole type should be chosen for perpendicular magnetic recording. Finally, there is even an opinion that the introduction of perpendicular magnetic recording has no merit no longer.

Indubitably, there are a lot of unsolved problems peculiar to perpendicular magnetic recording, i.e. the media noise, the head induced erasure and so on [2]. On the other hand, it is also true that a lot of glamorous characteristics of extremely high density recording are observed in laboratories $[3,4]$ and even the possibility of $100 \mathrm{~Gb} / \mathrm{inch}^{2}$ recording was confirmed by computer simulation [5].

The purpose of this paper is to discuss how does the next generation of magnetic recording build up. Various efforts are being carried out at present to develop an extremely high density recording system over $10 \mathrm{~Gb} /$ inch $^{2}$ for the next century. In this paper, first of all, we reconfirm analytically the reason why perpendicular magnetic recording is promising for extremely high density recording, though the purpose is not only to emphasize the advantage of perpendicular magnetic recording. Next, writing heads which are desirable for perpendicular magnetic recording are discussed on the premise that MR or GMR heads will be used from now on, and the distinctive features and writing characteristics of a novel single-pole type thin film head made as a trial are introduced. After that, three major technical problems will be discussed for practical use of perpendicular magnetic recording which are; media noise, thermal relaxation and head induced erasure.

\section{WRITING RESOLUTION}

The current high linear bit density has been won through the war against a demagnetizing field in magnetic recording. In longitudinal recording, it has been achieved by decreasing the thickness of the recording layer and increasing the coercive force. We confirmed by a computer simulation that a higher $\mathrm{H}_{c}$ brings both a higher resolution and a higher readout voltage in longitudinal recording [6]. A smaller $M_{r} \delta$ brings a higher writing resolution, but it also brings a lower readout voltage.

Meanwhile, for perpendicular magnetic recording using a single-pole head and a double-layered medium, we also found out by the simulation that the sharpness of the magnetic field distribution of the head and the gradient of the hysteresis curve of the media contribute to both a higher writing resolution and a higher readout voltage [6]. First of all, we estimate transition lengths in perpendicular magnetic recording by using an analytical model. 


\section{Isolated transition}

The distribution of perpendicular magnetization in the recording layer of a medium is self-consistently given by

$$
M_{y}(x)=M_{y}\left(H_{y}(x)\right) \quad(1-a)
$$

and

$$
\mathrm{H}_{\mathrm{y}}(\mathrm{x})=\mathrm{H}_{\mathrm{hy}}(\mathrm{x})+\mathrm{H}_{\mathrm{dy}}(\mathrm{x}) \quad(1-\mathrm{b}),
$$

where $M_{y}\left(H_{y}\right)$ is the dependence of the magnetization upon a magnetic field applied perpendicular to the medium and is given by the major hysteresis curve around the coercive force. $H_{h y}(x)$ and $H_{d y}(x)$ are the distributions of the head field and the demagnetizing field in the recording layer.

Now we assume that the recording layer consists of fine magnetic particles with spontaneous magnetization $I_{s}$ having unidirectional anisotropy. If the particles are perfectly oriented perpendicular to the surface of the medium, we can easily draw the hysteresis curve as the sum of the magnetization of each of the particles.

Furthermore, we assume that the distribution function, $\phi\left(h_{c}\right)$, of the coercive force of the magnetic particles composing the recording layer can be written as,

$$
\phi\left(h_{c}\right)=\frac{N}{\pi \Delta H_{c}} \frac{1}{1+\left(\frac{h_{c}-H_{c}}{\Delta H_{c}}\right)^{2}}
$$

This equation expresses the ratio of the number of particles, $\mathrm{n}$, whose coercive force is $\mathrm{h}_{\mathrm{c}}$, to the total particle number $\mathrm{N}$, per unit volume. The coercive force, $h_{c}$, of particles distributes with a half width of, $\Delta H_{c}$, around the average coercive force, $\mathrm{H}_{c}$, of the medium. Here we have defined that $\Delta \mathrm{H}_{c}$ must be smaller than $\mathrm{H}_{c} / 3$ so that the number of particles of $h_{c}=0$ becomes less than $10 \%$ of the total. For example, if $H_{c}$ is $1200 \mathrm{Oe}, \Delta H_{c}$ must be less than $400 \mathrm{Oe}$.

If a magnetic field, $\mathrm{H}_{\mathrm{y}}$, is applied perpendicular to a medium in the plus direction after all particles have been magnetized to the minus direction, the particles whose coercive force is $-\infty<h_{c} \leq H_{y}$ reverse this magnetization direction to the plus. Then the total magnetization is given by

$$
\mathrm{M}_{\mathrm{y}}(\mathbf{x})=\mathrm{I}_{\mathrm{s}} \int_{-\infty}^{\mathrm{HY}} \phi\left(\mathrm{h}_{\mathrm{c}}\right) \mathrm{d} \mathrm{h}_{\mathrm{c}}-\mathrm{I}_{\mathrm{s}} \int_{\mathrm{Hy}}^{\infty} \phi\left(\mathrm{h}_{\mathrm{c}}\right) \mathrm{d} \mathrm{h}_{\mathrm{c}}
$$

Meanwhile, the saturation magnetization $\mathrm{M}_{\mathrm{s}}$ is given by

$$
\mathrm{M}_{s}\left(\mathrm{H}_{\mathrm{y}}=\infty\right)=\mathrm{I}_{\mathrm{s}} \int_{-\infty}^{\infty} \phi\left(\mathrm{h}_{\mathrm{c}}\right) d \mathrm{dh}_{\mathrm{c}}=\mathrm{I}_{\mathrm{s}} \mathrm{N}
$$

because the saturation magnetization $\mathrm{M}_{\mathrm{s}}$ is the state in which all particles are magnetized in the same direction. Therefore, from equations (2) and (3), the major hysteresis curve from minus saturation $\mathrm{M}_{\mathrm{s}}$ when the plus field $\mathrm{H}_{\mathrm{y}}$ is applied perpendicular to the medium is given by

$$
\mathrm{M}_{\mathrm{y}}\left(\mathrm{H}_{\mathrm{y}}\right)=\frac{2 \mathrm{M}_{\mathrm{s}}}{\pi} \arctan \left(\frac{\mathrm{H}_{\mathrm{y}}-\mathrm{H}_{\mathrm{c}}}{\Delta \mathrm{H}_{\mathrm{c}}}\right)
$$

If particles are perfectly oriented in the direction perpendicular to the surface and magnetostatic interaction dose not occur between each particle, the residual magnetization $M_{r}$ becomes equal to the saturation magnetization $M_{s}$, i.e. $M_{r} / M_{s}=1$.

Now, if a head field distribution around the position $\mathrm{x}_{0}$, where $H_{h y}\left(x_{0}\right)=H_{c}$, can be approximated as a straight line of slope $\alpha$, it can be expressed by the following equation,

$$
\mathrm{H}_{\text {hy }}(\mathrm{x})=\mathrm{H}_{\mathrm{c}}-\alpha\left(\mathrm{x}-\mathrm{x}_{\mathrm{o}}\right)
$$

By substituting equation (4) and (6) for (1), a selfconsistent magnetization distribution in a perpendicular recording layer is obtained as follows,

$$
\operatorname{My}(\mathbf{x})=\frac{2 \mathrm{M}_{\mathrm{r}}}{\pi} \arctan \left\{\frac{-\alpha\left(\mathrm{x}-\mathbf{x}_{\mathrm{o}}\right)+\mathrm{H}_{\mathrm{dy}}(\mathbf{x})}{\Delta \mathrm{H}_{\mathrm{c}}}\right\}
$$

where $M_{r} / M_{s}=1$.

Consequently, an isolated transition of perpendicular magnetization is determined by not only the field gradient $\alpha$ and the particle coercive force distribution $\Delta H_{c}$, but also the perpendicular demagnetizing field distribution, $\mathrm{H}_{\mathrm{dy}}$.

\section{Demagnetizing field}

Now we approximate a perpendicular magnetization distribution of half transition length, a, in the recording layer of a medium by the following arctangent function,

$$
\mathbf{M}_{\mathbf{y}}(\mathbf{x})=\frac{2 \mathrm{M}_{\mathrm{r}}}{\pi} \arctan \left(\frac{\mathrm{x}}{\mathrm{a}}\right)
$$

as shown by the dotted curve in Fig. 1.

If the origin of the $y$ axis is put on the middle plane of the recording layer and the existence of the magnetic 
charges on the top and bottom surfaces of the recording layer is represented by the constants $\mathrm{K}_{\mathrm{t}}$ and $\mathrm{K}_{\mathrm{b}}$ respectively, the distribution of the demagnetizing field in the recording layer of thickness $\delta$ is given as follows [7],

$$
\begin{aligned}
\mathrm{H}_{\mathrm{dy}}(\mathrm{x}, \mathrm{y}) & =-4 \mathrm{M}_{\mathrm{r}}\left\{\mathrm{K}_{\mathrm{t}} \arctan \left(\frac{\mathrm{x}}{\mathrm{a}+\frac{\delta}{2}-\mathrm{y}}\right)\right. \\
& \left.+\mathrm{K}_{\mathrm{b}} \arctan \left(\frac{\mathrm{x}}{\mathrm{y}+\frac{\delta}{2}+\mathrm{a}}\right)\right\}
\end{aligned}
$$

Here, $K_{t}=1$ and $K_{b}=1$ for a single-layered medium and $K_{t}=1$ and $K_{b}=0$ for a double-layered medium.

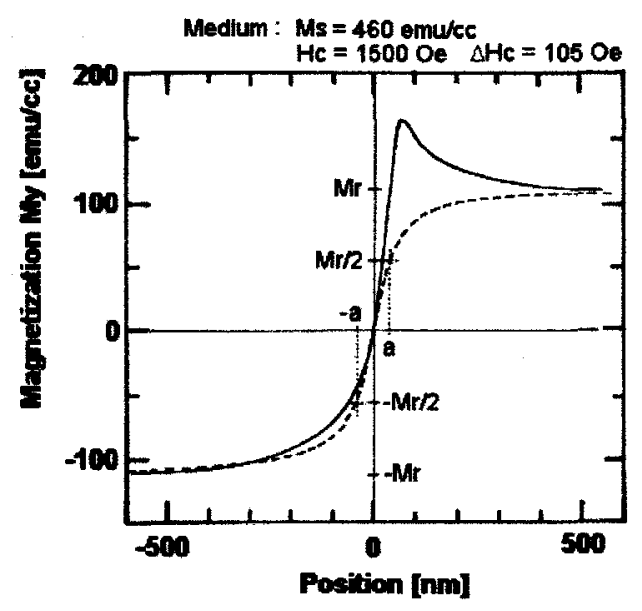

Fig. 1 Isolated transition magnetization distribution in a perpendicularly magnetized recording layer.

A read head detects the magnetic field generated from the charge on the top surface of the recording layer. Therefore, if the effect of a demagnetizing field upon the transition length of a perpendicular magnetization on the top surface of the recording layer is taken into account, the demagnetizing field is approximately given by

$$
H_{d y}\left(x, \frac{\delta}{2}\right)=\kappa M_{y}\left(x, \frac{\delta}{2}\right)
$$

Here $\kappa$ is $2 \pi$ for a double-layered medium and $4 \pi$ for a thinner single-layered medium.

\section{Transition length}

A half transition length, $a$, is defined as the distance from the transition center $x=x_{0}$ to the position where $M_{y}(x)$ is $\pm M_{r} / 2$, as expressed by equation (7) and (8). Therefore, by substituting equation (10) for equation (7) and moreover $x_{0}+a$ or $x_{0}-a$ for $x$, and $+M_{r} / 2$ or $-M_{r} / 2$ for $M_{y}(x)$ in equation (7), the half transition length, $a$, is given as

$$
\mathrm{a}=\frac{\Delta \mathrm{H}_{\mathrm{c}}}{\alpha}+\frac{\kappa \mathrm{M}_{\mathrm{r}}}{2 \alpha}
$$

The first term of the right hand side shows the intrinsic isolated half transition length, $a_{0}$, determined by the field gradient, $\alpha$, of the head and the dispersion, $\Delta \mathrm{H}_{\mathrm{c}}$, of particle coercive forces of the medium. The second term is the effect of the demagnetizing field applied just after the reversal of a head field.

Therefore, the demagnetizing field acts so as to spread the intrinsic isolated transition length, $2 a_{0}$, but at a higher bit density, the actual transition length, $2 a$, will decreases to the intrinsic value, $2 a_{0}$, regardless of a double- and a singlelayered media. This is because the demagnetizing coefficient, $\kappa$, will decrease to almost zero with increasing bit density. If we assume that $\mathrm{M}_{\mathrm{r}}$ of a medium is 400 $\mathrm{emu} / \mathrm{cc}, \Delta \mathrm{H}_{\mathrm{c}}$ is $100 \mathrm{Oe}$ for $\mathrm{H}_{\mathrm{c}}=2000 \mathrm{Oe}$ and $\alpha$ of a head is $20 \mathrm{Oe} / \mathrm{nm}$, the actual transition lengths, $2 \mathrm{a}$, are calculated as $136 \mathrm{~nm}$ and $262 \mathrm{~nm}$ respectively for a double- and a single-layered media, but the intrinsic transition length, $2 a_{0}$, is only $10 \mathrm{~nm}$.

Furthermore, after removal of the head field, the transition length, a, in longitudinal magnetic recording is widened further by the demagnetizing field. However, in perpendicular magnetic recording, we can disregard such a secondary effect of the demagnetizing field, because the demagnetizing coefficient of perpendicular magnetization is roughly assumed to be constant, independent of the distance, $\mathrm{x}$, from the transition center.

In perpendicular magnetic recording, the residual magnetization, $M_{r}$, between the transitions is decreased remarkably to $M_{r}^{\prime}=H_{c} / \kappa$, where $\kappa$ is $2 \pi$ for a double-layered medium and $4 \pi$ for a single-layered medium. Since the magnetization decreases gradually with approaching the transition center, $\mathrm{x}=0$, the demagnetizing field strength also decreases from the maximum value of $\mathrm{kM}_{\mathrm{r}}{ }^{\prime}$ to 0 with approaching the transition center. Therefore, the half transition length, $\mathrm{a}$, which is the distance, $\Delta \mathrm{x}$, from $\mathrm{x}=0$ to the position, $x$, where $M_{y}=M_{r}^{\prime} / 2$, will become rather short than that given by equation (11) when a medium having a higher $H_{c}$ and a narrower $\Delta H_{c}$ is used, because the demagnetizing field decreases remarkably to $\mathrm{H}_{\mathrm{c}} / 2 \mathrm{~K}$ at $\mathrm{x}=\mathrm{a}$. 
demagnetizing field decreases remarkably to $\mathrm{H}_{\mathrm{c}} / 2 \kappa$ at $\mathrm{x}=\mathrm{a}$.

Consequently, we have confirmed again that perpendicular magnetic recording undoubtedly has a possibility of extremely high bit density recording whose bit size corresponds to diameters of several magnetic particles when a sharper gradient, $\alpha$, of the perpendicular field distribution of the head and a narrower half-peakwidth, $\Delta H_{c}$, of the particle coercive force distribution of the medium are realized.

\section{WRITING HEAD}

From a view point of the sharpness of the head field distribution around the trailing edge, we discuss next about a writing head which is suitable for perpendicular magnetic recording.

\section{Writing field}

For a computation of the writing field, we have assumed for a single-pole head that the thickness of the main-pole is $200 \mathrm{~nm}$, the head-to-medium spacing is $20 \mathrm{~nm}$ and the thickness of the recording layer is $50 \mathrm{~nm}$. Both the distributions of the perpendicular and the longitudinal field components on the layer parallel to the top surface of the medium, which is $35 \mathrm{~nm}$ apart from the top of the mainpole, have been computed by a finite element method. For comparison, both the distributions of a ring head, whose gap length is $200 \mathrm{~nm}$, have been computed on the same layer.

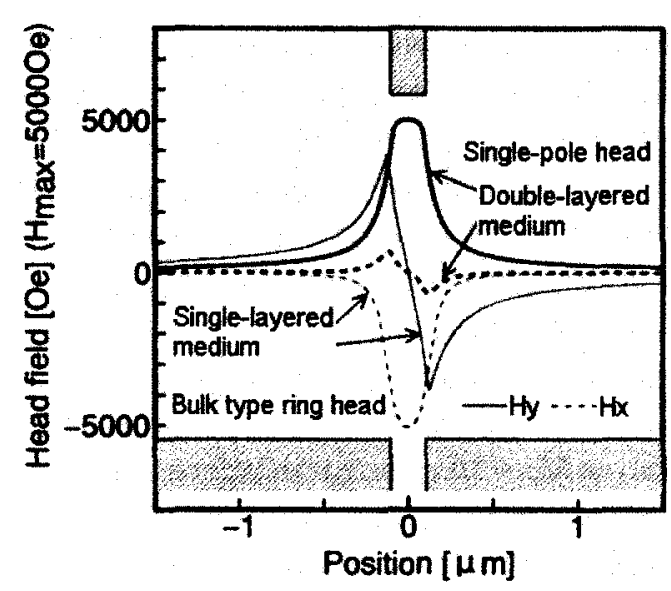

Fig. 2 Magnetic field distribution of a single-pole head and a ring head.

We can confirm that the perpendicular component of a single-pole head has a favorably steep distribution in comparison with the longitudinal component of a ring head (Fig. 2). For example, the maximum gradient of the former is $48.5 \mathrm{Oe} / \mathrm{nm}$, while the latter is $37.5 \mathrm{Oe} / \mathrm{nm}$, on the layer $35 \mathrm{~nm}$ apart from the head surface, when the maximum field strength of $5000 \mathrm{Oe}$ is applied on the same layer. Furthermore, we can see that the longitudinal component of the single-pole head is considerably smaller than the perpendicular component of the ring head.

Perpendicular magnetic recording is also made possible by the perpendicular component of a ring head field. But it spreads widely and decreases gently over the area facing a medium in a bulk type ring head (Fig. 2). Therefore the isolated transition is widened, so that serious interference is occurred between each transition at a higher bit density. To avoid this, a narrower gap is necessary and indispensable for reducing the field distribution area [8]. A narrower particle coercive force distribution of media and so a higher rectangularity is also efficient.

Recently, excellent bit density characteristics have been obtained by using thin-film type ring heads. In such a head, a similar sharpness to a single-pole head is produced around the trailing edge of the upper core (trailing core) because the thickness of the trailing core is restricted by the film thickness (Fig. 3). This phenomenon is especially remarkable when a double-layered medium with a soft magnetic under-layer is combined with the head. For example, we can compute a maximum field gradient of 48 $\mathrm{Oe} / \mathrm{nm}$ around the trailing edge of the trailing core under the same condition as that computed for a single-pole and a ring head (Fig. 2).

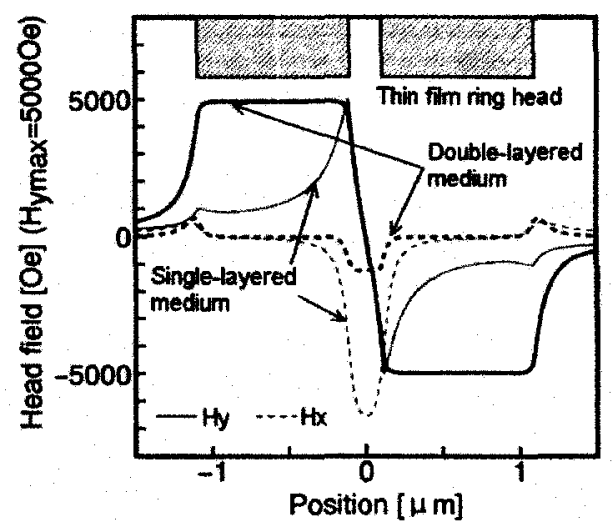

Fig. 3 Magnetic field distribution of a thin-film type ring head for a single- and a double-layered medium.

However, if a single-layered medium is combined with the head, the distribution of the perpendicular component reduces gently around the leading edge of the trailing core like a ring type bulk head, because of a weaker head-tomedium interaction (Fig. 3). Therefore, a narrower gap and a thinner trailing core of the head and a higher 
density recording.

Consequently, a single-pole head combined with a double-layered medium is the most suitable for ultra-high density recording. A ring type thin film head also has a potential as a writing head for perpendicular magnetic recording but a little modification of the structure may be necessary.

\section{Recording layer effect}

The sharpness of the magnetic field around a single-pole head depends on the magnetic interaction with the medium [9]. To make the head-to-medium magnetic interaction stronger, a narrower separation between the main-pole of the head and the soft magnetic backing layer of the medium is effective. If the head-to-medium spacing is constant, therefore, it is desired that the recording layer is relatively thin compared with the thickness of the main-pole. A similar effect is also expected by increasing the saturation magnetization of the recording layer.

To confirm this effect, the half transition length, $a$, in the distribution of magnetic flux density on the surface of a medium, after writing an isolated transition, has been investigated by the magnetic recording simulator (Fig. 4). The simulation has been carried out for various recording layer thicknesses from 10 to $250 \mathrm{~nm}$ under a fixed mainpole thickness of $200 \mathrm{~nm}$ and head-to-medium spacing of $20 \mathrm{~nm}$. Moreover, we have assumed that the recording layer has a coercive force, $\mathrm{H}_{c}$, of $1500 \mathrm{Oe}$, a particle coercive force dispersion, $\Delta \mathrm{H}_{\mathrm{c}}$, of $105 \mathrm{Oe}$ and a saturation magnetization of $460 \mathrm{emu} / \mathrm{cc}$.

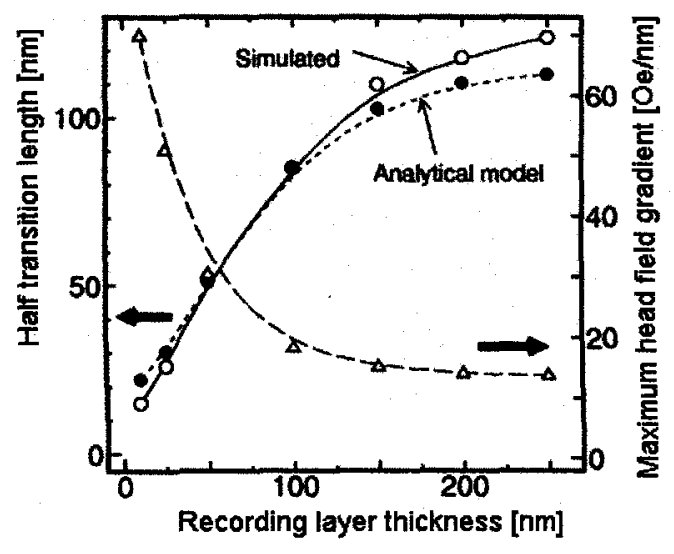

Fig. 4 Medium thickness dependency of isolated half transition length in perpendicular magnetic recording.

The results have been compared with those calculated by using the above mentioned analytical model of equation (11) (Fig. 4). The field gradients of the single-pole head used for the analyses are those on the layer near the bottom surface of a recording layer, which is computed by the finite element method in taking no account of the existence of the recording layer. The results clearly show that the analytical model is useful for a rough estimation of the transition length in perpendicular magnetic recording.

On the other hand, from the computer simulation, we can see that the maximum magnetic flux density on the medium surface increases with increasing recording layer thickness (Fig. 5), but the transition width is widened because of a decrease in the magnetic coupling with the soft magnetic backing layer. At a higher bit density, therefore, the amplitude of the surface flux density increases with increasing recording layer thickness for a relatively thin recording layer, but decreases as the recording layer thickness is increased from the optimum thickness. The optimum thickness is given by the thickness at which the transition length, 2a, becomes almost the same as a bit length (Fig.5). At the optimum thickness, therefore, the writing resolution is improved but the surface flux density decreases at a lower bit density.

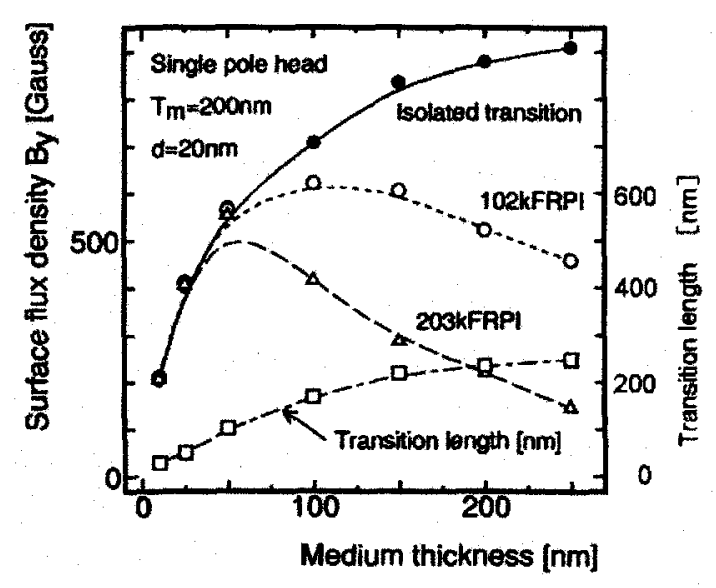

Fig. 5 Medium thickness dependency of the amplitude of the surface flux density for a various bit density obtained by the simulation.

\section{NOVEL SINGLE-POLE HEAD}

The most practical candidate for a writing head for perpendicular magnetic recording is a ring type inductive thin film head which is merged with a MR read head used for the current HDD. However, for practical use, the trailing edge of the trailing core should be carefully manufactured because the transitions of perpendicular magnetization are written here, especially for a double layered medium.

On the other hand, we have made the novel practical 
On the other hand, we have made the novel practical single-pole type thin film flying head as a trial (Fig. 6), in cooperation with Sony corporation. The experimental single-pole heads have been made so as to directly energize the top of a main-pole film, which is sandwiched between thin film conductor strips of several hundreds nano-meter in thickness. They are loaded onto a nano-slider whose flying height is about $40 \mathrm{~nm}$.

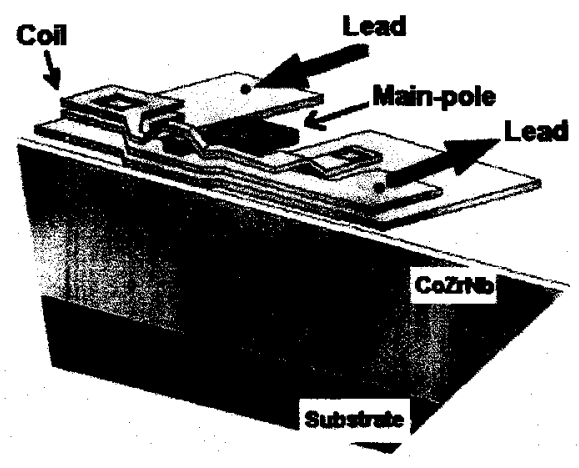

Fig. 6 Structure of the novel thin-film type single-pole head for a low flying head.

The structure of this head is simpler than that of a conventional ring type thin film head, so that it can be made easily by a conventional production process of thinfilm heads. We have confirmed with the simulator that the slope of the perpendicular field distribution is almost the same as that of a bulk type single-pole head.

The writing characteristics of this type of head have been measured by using a MR read head (Fig. 7 and 8). The shield gap length of a MR head has been estimated from the bit density characteristics at roughly $200 \mathrm{~nm}$. The disk used for the measurement was a three-layered medium produced by JVC, which consists of a $50 \mathrm{~nm}$ thick CoCrTa recording layer, a $600 \mathrm{~nm}$ thick $\mathrm{CoZrNb}$ soft magnetic backing layer and a $150 \mathrm{~nm}$ thick SmCo hard magnetic layer [10]. The magnetic domains of the $\mathrm{CoZrNb}$ soft magnetic layer are controlled by the SmCo hard magnetic layer. The $\mathrm{M}_{\mathrm{s}}, \mathrm{H}_{\mathrm{c}}$ and $\Delta \mathrm{H}_{\mathrm{c}}$ of the CoCrTa layer were 500 $\mathrm{emu} / \mathrm{cc}, 2000 \mathrm{Oe}$ and $100 \mathrm{Oe}$, respectively. For comparison, a ring type thin film head has been measured in combination with a longitudinal hard disk of $100 \mathrm{G} \mu \mathrm{m}$ in $\mathrm{B}_{\mathrm{r}} \delta$ and 2400 Oe in $\mathrm{H}_{\mathrm{c}}$.

The writing resolution $\mathrm{D}_{50}$ of the novel single-pole thin film head was about $160 \mathrm{kFCI}$, which is slightly lower than $170 \mathrm{kFCI}$ of the current bulk type single-pole head for the same medium (Fig. 7). Meanwhile, in longitudinal recording measured with the same MR head, $D_{50}$ was about $130 \mathrm{kFCl}$. Writing resolutions and readout voltages are roughly 1.3 times and 1.7 times higher respectively in comparison with longitudinal recording.

It is clear that the bit density response in perpendicular magnetic recording is restricted by the shield gap length of the MR read head because we have already observed a readout voltage, even at bit densities over $500 \mathrm{kFCI}(20$ $\mathrm{kFCM}$ ), with a single-pole inductive head. Therefore, a narrower shield gap in the MR head would produce a higher bit density response.

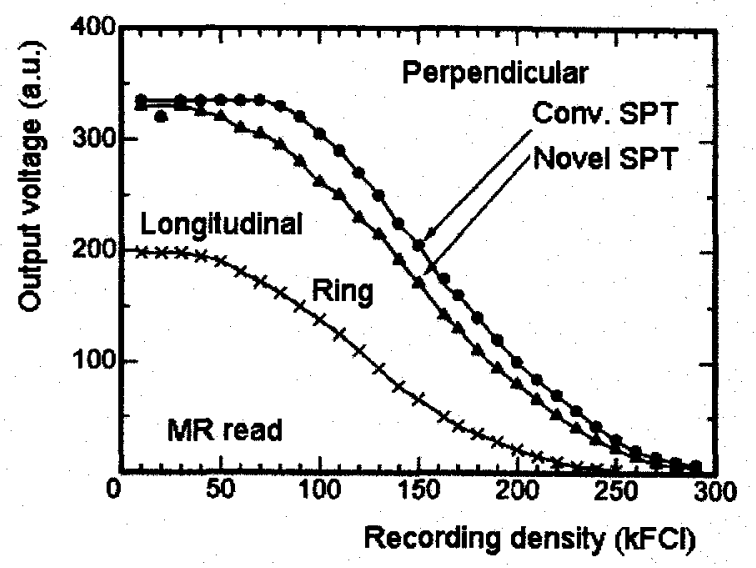

Fig. 7 Bit density characteristics of the novel thin film type single-pole head in comparison with a conventional bulk type single-pole head and a thin film type ring head.

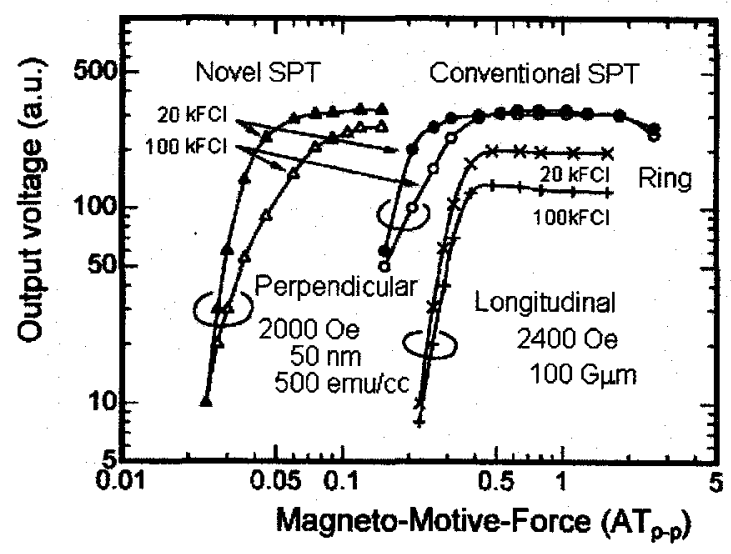

Fig. 8 Writing characteristics of the novel thin film type single-pole head in comparison with a conventional bulk type single-pole head and a thin film type ring head.

In a MR head readout, the voltage waveform is almost the same as the perpendicular magnetization distribution on the top layer of the medium. It was already reported that a single peak pulse becomes similar to that in longitudinal recording by the differentiation of MR read voltage [11]. 
Though the peak voltage is reduced, the half pulse width is also reduced, and then $\mathrm{D}_{50}$ is improved remarkably from $170 \mathrm{kFCI}$ to $270 \mathrm{kFCI}$. Moreover, a media noise peculiar to perpendicular magnetic recording, observed in a lower frequency band, is suppressed by the differentiation. Recently a relatively lower error rate has been reported by using a differential method [12].

The most important feature of the novel head is that the writing magneto-motive force is remarkably small (Fig. 8). This is roughly one fifth of that of the current bulk type single-pole head, with the same perpendicular disk, and one tenth of that of the ring type thin film head with a longitudinal hard disk. This is just an effect of directly energizing the main-pole top due to the thin film conductors. The other important feature is that the inductance is much smaller than $10 \mathrm{nH}$, so that this head is expected to find high data rate applications.

\section{TECHNICAL ISSUES}

Some of technical issues for the practical use of perpendicular magnetic recording are common to those of higher density longitudinal recording, but media noise and domain control for soft magnetic films of the head and medium are serious problems. As a result of many efforts to solve these, the technological gap with longitudinal recording is closing step by step.

\section{Media noise}

In perpendicular magnetic hard disks, a larger media noise is observed at a lower bit density. Therefore it has been presumed that medium noise in perpendicular recording media arises from local reversal domains which are magnetized in opposition to the surrounding area which is otherwise magnetized uni-directionally between each transition (Fig. 9) [13]. The fluctuations of the size and the density of local reversal domains cause a serious noise, so that the media noise decreases with increasing bit density because the DC magnetized area between each transition narrows.

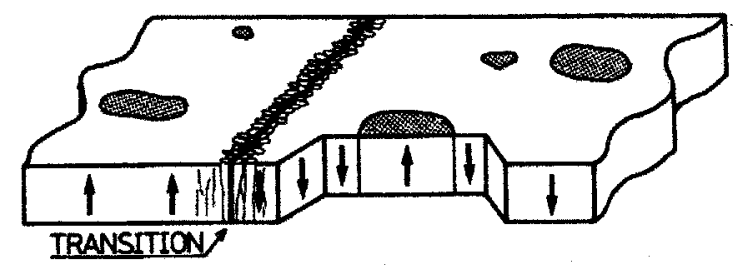

Fig. 9 Local reversal domain model in a perpendicularly magnetized medium.
A local reversal domain in a recording layer of a perpendicular magnetic recording medium may be a cluster of particles magnetized in opposition to the magnetization of the surrounding particles. When a recording layer of the saturation magnetization, $\mathrm{M}_{\mathrm{s}}$, consists of magnetic particles of spontaneous magnetization, $I_{s}$, but their coercive forces, $h_{c}$, are dispersed in the film plane, the magnetization direction of particles whose $h_{c}$ is smaller than the perpendicular demagnetizing field strength, $\mathrm{H}_{\mathrm{dy}}=4 \pi \mathrm{M}_{\mathrm{s}}$, should be reversed.

If the particles of $h_{c}<H_{d y}$ are gathered together with an exchange interaction, the magnetization should be reversed in clusters and this should create larger local reversal domains. To avoid this, higher $h_{c}$ of particles and smaller $M_{s}$ of the medium are required so as to make $h_{c} / M_{s}>4 \pi$. Not only a smaller size and a narrower $h_{c}$ dispersion of particles, but also more isolated particles and a weaker exchange interaction will be required for lower noise in a perpendicular recording medium.

It is well known empirically that medium noise in perpendicular magnetic recording decreases in proportion to $\mathrm{M}_{\mathrm{s}} \delta\left(1-\mathrm{M}_{\mathrm{r}} / \mathrm{M}_{\mathrm{s}}\right)$ for a $\mathrm{Co}-\mathrm{Cr}$ system recording layer [14]. When the coercive force of the medium is $\mathrm{H}_{c}$ and it corresponds to the average of particle coercive forces, $h_{c}$, local reversal domains in the residual magnetization state reduce the net residual magnetization $M_{\tau}$ to $H_{c} / 4 \pi$. Then the hysteresis loop is tilted. Therefore, when $M_{r} / M_{s}$ approaches 1 by decreasing $M_{s}$ or increasing $H_{c}$, media noise can be decreased.

However, even if $M_{r} / M_{s}$ is made nearly 1 , a relatively high medium noise still occasionally remains. The reason is speculated that the noise will come from small fluctuation in the magnetization direction of particles, thickness of the recording layer, volume of particles and so on, because these cause a fluctuation of the magnetic flux density on the medium surface. If these parameters fluctuate more than $3 \%$, for example a magnetization direction more than 14 degree or a surface roughness of more than $1.5 \mathrm{~nm}$ for a $500 \mathrm{~nm}$ thick recording layer, it may cause a medium noise of more than $30 \mathrm{~dB}$ in $\mathrm{S} / \mathrm{N}$.

\section{Thermal relaxation}

We have theoretically investigated the thermal relaxation in perpendicular magnetic recording by a computer simulation [15]. In the simulation, we assumed that the recording layer consists of particles taking a curling incoherent mode of the magnetization reversal. If the spontaneous magnetization, the unidirectional anisotropy and the volume of a particle are given by $I_{s}, K_{w}$, and $V$, 
respectively, and a critical field, $\mathrm{H}_{n}$, is required for the reversal of the particle, the magnetic energy can be given by $E_{m}=I_{s} H_{n} V$ for the reversal, instead of $K_{a} V$.

Then, assuming the temperature, $T$, and giving the Boltzmann constant, $k$, the relaxation time, $\tau$, is given by

$$
\tau=10^{-9} \exp \left(\frac{\mathrm{H}_{\mathrm{n}} \mathrm{I}_{\mathrm{s}} \mathrm{V}}{\mathrm{kT}}\right)
$$

Therefore, when a magnetic field $\mathrm{H}$ is applied to a particle whose magnetization direction is $\phi$, the relaxation time is given as follows,

$$
\tau=10^{-9} \exp \left\{\frac{\left(\mathrm{H}_{\mathrm{n}}-\mathrm{H} \cos \phi\right) \mathrm{I}_{\mathrm{s}} \mathrm{V}}{\mathrm{kT}}\right\}
$$

The equation means that the energy barrier which will be overcome by the thermal energy $E_{t}=k T$ is reduced to $\left(H_{n}-\right.$ $H \cos \phi$ ) by applying the reverse field $H$.

In the simulation, the relaxation times, $\tau$, for every particle at every cell are given by equation (13), replacing $\mathrm{H}$ with a demagnetizing field $\mathrm{H}_{\mathrm{d}}$ calculated from the magnetization distribution. Then, the magnetization distribution, reduced by the thermal relaxation after a time interval of $\Delta t$ seconds, has been self-consistently computed by using the following equation,

$$
M(t)=I_{s} \exp \{-\Delta t / \tau\}
$$

This procedure has been repeated every $\Delta t$ seconds.

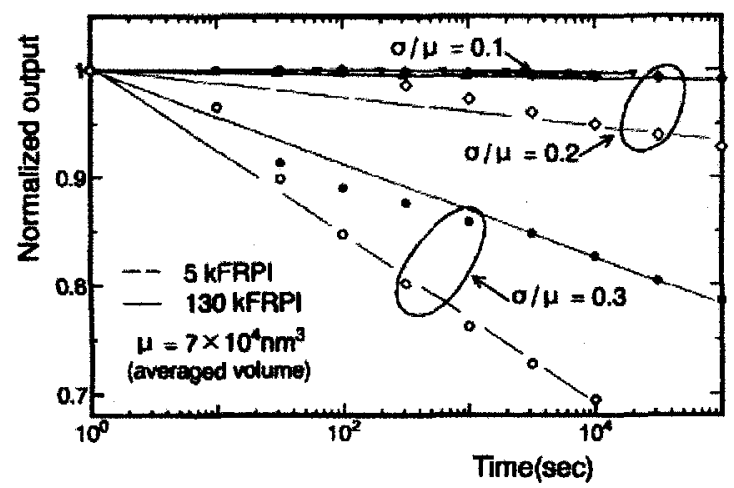

Fig. 10 Thermal relaxation simulation for perpendicular magnetic recording media. ( $\mu$ : average volume of particle, $\sigma:$ standard deviation of particle volume).

We have confirmed by the simulation that a larger thermal relaxation occurs, even in perpendicular magnetic recording, when the recording layer consists of very smaller particles with a very wide dispersion sizes (Fig. 10). But it becomes considerably reduced at a higher bit density. This is the completely the opposite to longitudinal recording.

In experiments, we have clearly observed that the readout voltage decreases with passing time, especially at a lower bit density and when writing and reading have been carried out by using the same single-pole head (Fig. 11). But, when a single-pole head has been unloaded after writing and only a MR head is loaded for reading, a reduction has not been observed in readout voltage even at a lower bit density.

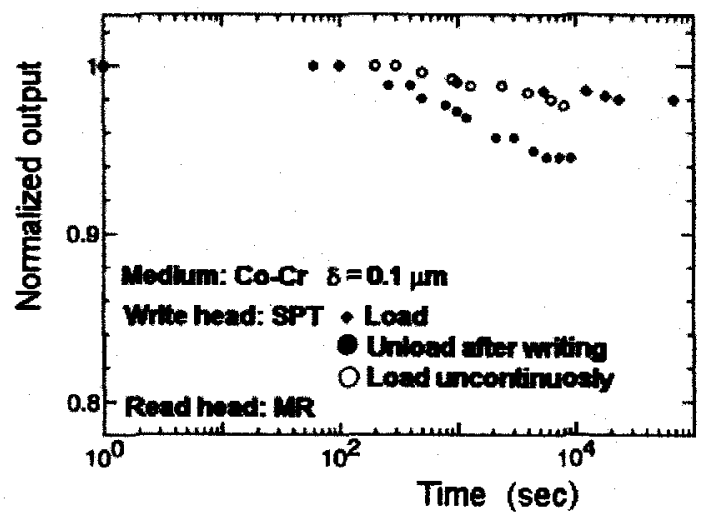

Fig. 11 Reduction of readout voltage due to loading of a bulk type conventional single-pole head.

Consequently, we can conclude that the thermal relaxation effect is hardly observed in perpendicular magnetic recording, especially at a higher bit density. However a serious reduction of the readout voltage caused from head induced erasure may be observed when a singlepole writing head is loaded.

\section{Head induced erasure}

It was reported for perpendicular magnetic recording that the readout voltage is not only decreased gradually with passing time but also erased locally when a single-pole head is combined with a double layered medium [2]. For the latter, a domain control for a soft magnetic backing layer of a medium is very useful, in which the exchange interaction effect of a $\mathrm{SmCo}$ hard magnetic layer is utilized [10]. This is because the local erasure is probably caused by an instantaneous movement of a domain wall.

Meanwhile, it is supposed that the former is caused from a stray field gathering effect of a single-pole head. If magnetic domains around the top of a main-pole have the perpendicular magnetization component, a weak magnetic field is applied perpendicular to the medium, even in a 
reading state, so that the thermal relaxation is accelerated by this field and the data written in the disk may be gradually erased.

We have experimentally confirmed that a single-pole head is more sensitive than a ring head to the perpendicular component of a stray. field (Fig. 12). A perpendicular recording layer is also more sensitive than a longitudinal recording media when a ring type thin film head is used. Meanwhile, the novel single-pole type thin film head developed by us is much less sensitive than a conventional ring type thin film head to the stray field gathering effect.

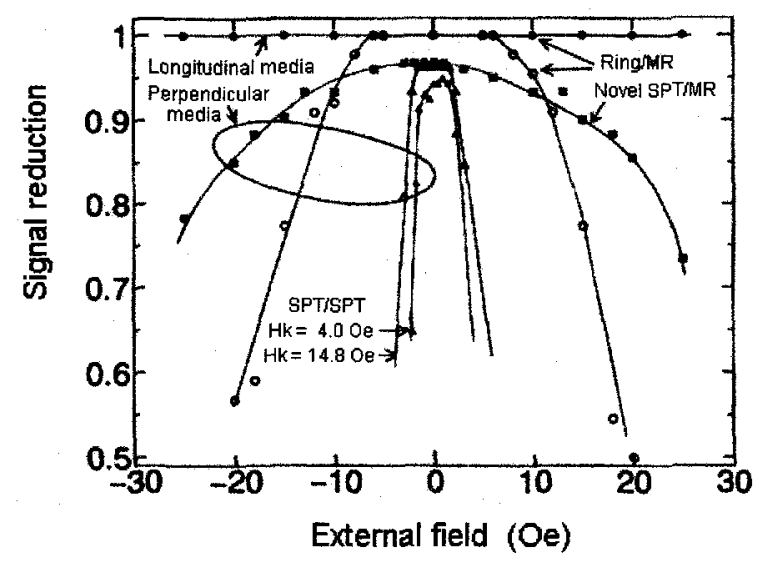

Fig. 12 Signal reductions of various types of heads caused by perpendicular stray field.

However, even if no stray field is applied to a head, the reduction of the readout voltage is still observed slightly in a single-pole head. This may be due to wall instability in the main-pole and weak thermal relaxation of the perpendicular recording layer at a lower bit density, which comes from imperfections of the medium or the head. Therefore, it is expected that head induced erasure may scarcely occur when a main-pole film made of a higher $H_{k}$ or a multi-layered film is introduced for the novel thin film single-pole head.

Consequently, to prevent head induced erasure, the stabilization of domain structures in the soft magnetic backing layer of the medium and the main-pole film of the head is the most important subject.

\section{100 GBPSI SIMULATION}

Finally we have investigated the possibility of 100 Gbit/inch $^{2}$ by using the simulator and the necessary conditions for writing head and media to make 100 Gbit/inch ${ }^{2}$ recording possible [5].

In PMRC '94, we proposed a method for deep sub- micron track width recording, in which a data track is written without any attenuation of field strength by a mainpole thickness writing of a single-pole head $[16,17,18]$. We confirmed experimentally by a magneto-motive force microscopic observation that data of $13 \mathrm{Gbit} / \mathrm{inch}^{2}$ is able to be written clearly at a track width of $0.4 \mu \mathrm{m}$.

In the magnetic recording simulator, we have assumed that a data track is written by a $75 \mathrm{~nm}$ thick main-pole of a single-pole head. For the recording layer, we have also assumed that columnar particles of $12.5 \mathrm{~nm}$ in diameter and 1 to 4 aspect ratio are systematically arranged on a substrate and that a moderate exchange interaction acts among particles.

The coercive force and easy magnetization direction of each particle at each mesh point have been determined by a table of random number according to the probability distributions. Both distributions of the coercive force and easy magnetization direction have been given by Gaussian functions and their standard deviations have been chosen relatively narrower values from the measured ones. Moreover the interparticle exchange interaction has been taken into account with the equivalent mean field. Using the equivalent mean field, a similar hysteresis loop to the measured one can be reconstructed by computer [5].

We have confirmed that data at more than $100 \mathrm{Gbit}$ inch $^{2}$ density $\left(160 \mathrm{Mbit} / \mathrm{mm}^{2}\right)$ can be written under these conditions at a track pitch of $125 \mathrm{~nm}$ with relatively good $\mathrm{S} / \mathrm{N}$, track densities of $20 \mathrm{kTPI}(0.8 \mathrm{kTPM})$ and linear bit density of $400 \sim 500 \mathrm{kFCI}(16 \sim 20 \mathrm{kFCM})$ ) (Fig. 13).

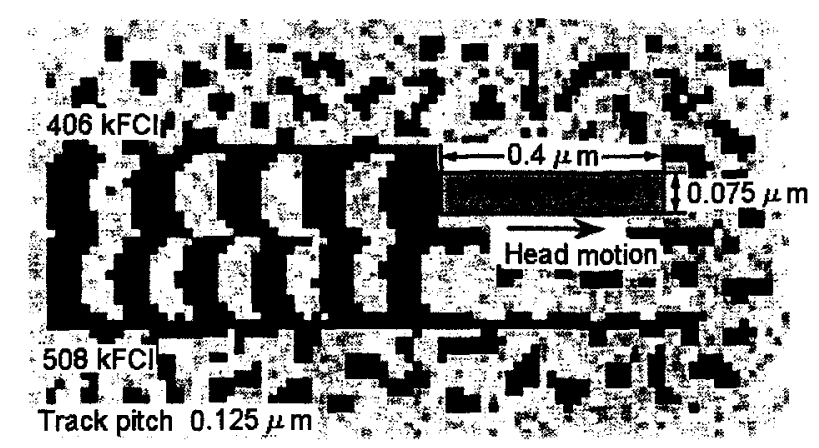

Fig. 13 Writing pattern of $100 \mathrm{~Gb}$ inch $^{2}$ data on a double layered medium after a main-pole thickness width writing by a single-pole head.

\section{CONCLUSION}

We can confirm that perpendicular magnetic recording has an enormous potential for ultrahigh density recording at more than $100 \mathrm{Gbpsi}$. On the other hand, we know that perpendicular magnetic recording has a lot of peculiar 
problems for practical use. To exceed $10 \mathrm{Gbit} / \mathrm{inch}^{2}$, several technological breakthroughs will be required in more accurate head and media manufacturing processes, including domain control technologies, tracking servo technology for deep-submicron width tracks, signal processing for an ultrahigh density and a higher transfer rate and so on

Above all, more practical media and heads must be indispensable to examine practically perpendicular magnetic recording. We still believe that perpendicular magnetic recording is a powerful candidate for ultrahigh density magnetic recording.

The author wishes to thank JVC Corp. and Sony Corp. for their cooperation, Prof. Dr. Y. Sugita and Associate Prof. Dr. H. Muraoka for their valuable discussions and Mr. Yamada, Ms. W. H. Jiang, Mr. T. Sasaki for their help in experiments and simulations.

This research partly supported by Storage Research Consortium, Association of Super-Advanced Electronics Technologies, and Grant-in Aid for Scientific Research of the Ministry of Education, Science and Culture.

\section{REFERENCES}

[1] S. Iwasaki and Y. Nakamura, "An analysis for the magnetization mode for high density magnetic recording," IEEE Trans. Magn., vol. MAG-13, pp.1272-1277, 1977.

[2] W. Cain, A. Payne, M. Baldwinson and R. Hempstead, "Challenges in practical implementation of perpendicular magnetic recording," IEEE Trans. Magn., vol. 32, pp.97$102,1996$.

[3] S. Yamamoto, Y. Nakamura and S. Iwasaki, "Extremely high bit density recording with single-pole perpendicular head," IEEE Trans. Magn., vol. MAG-23, pp.2070-2072, 1987.

[4] N. Honda. K. Ouchi and S. Iwasaki, "Extremely high linear density recording by perpendicular magnetization., vol. 32 , pp.3804-3806, 1996.

[5] Y. Nakamura, I. Tagawa and Y. Shimizu, "Ultra-high bit density magnetic recording -- Estimation based on three dimensional simulation --," Trans. IEICE Jpn., vol. J79-C-1, pp.152-164, 1996 (in Japanese).

[6] Y. Nakamura, "Theoretical and experimental prospects of perpendicular magnetic recording," Jour. MSJ Jpn., vol. 15 Supplement, pp.497-506, 1991.

[7] B. K. Middleton, "Chap.2 Recording and reproducing process," Magnetic recording technology edited by C. D. Mee and E. D. Daniel, pp.2.1-2.72. 1995.
[8] S. Onodera, S. Okijima, K. Ouchi, Y. Nakamura and S. Iwasaki, "Co-Cr perpendicular hard disk media with thin Fe-Co backlayer for ring-type head," Jour. MSJ Jpn., vol. 15 Supplement, pp.287-292, 1991.

[9] Y. Nakamura, S. Yamamoto and S. Iwasaki, "The effect of magnetic interaction between medium and head on perpendicular magnetic recording characteristics," IEEE Trans. Magn., vol. MAG-22, pp.376-378, 1986.

[10] T. Ando, M. Mizukami and T. Nishihara, "Effects of inplane hard magnetic layer on demagnetization and media noise in triple-layered perpendicular recording media," IEICE Trans. Elec., vol. E78-C, pp.1543-1549, 1995.

[11] H. Muraoka, H. Yamada and Y. Nakamura, "MR head reading characteristics in perpendicular magentic recording," IEEE Trans. Magn., vol. 32, pp.3482-3484, 1996.

[12] K. Ho, M. H. Kane, B. M. Lairson, G. I. Noynes, J. C. Harig and Y. K. Kim, "Data storage and retrieval using perpendicular media and magnetoresistive read transducer," IEEE Trans. Magn., vol. 33, pp.2538-2542, 1997.

[13] Y. Sano, H. Muraoka, I. Watanabe and Y. Nakamura, "Effects of nitrogen addition to the $\mathrm{CoCr}$ recordig layer of a double layered perpendicular rigid disk," Jour. MMM, vol. 155, pp.212-215, 1996.

[14] S. Yanase, L. Wo, N. Honda and K. Ouchi, "Recording and noise properties of perpendicular media for ring head," Proc. $5^{\text {th }}$ PMRS, pp.164-167, 1996. (in Japanese)

[15] W. H. Jiang, H. Muraoka, Y. Sugita and Y. Nakamura, "Thermal relaxation in perpendicular recording with single-pole head and MR head," Proc. $4^{\text {th }}$ PMRC (to be presented), 22aA-9, 1997.

[16] Y. Nakamura, "Challenge to terabit perpendicular spinic storage," Jour. HSJ Jpn, vol. 18 Supplement, pp. 161-170, 1994.

[17] H. Muraoka and Y. Nakamura, "Multi-track submicronwidth recording with a novel integrated single pole head in perpendicular magnetic recording," IEEE Trans. Magn., vol. 30, pp.3900-3902, 1994.

[18] Y. Nakamura, "Extremely high-density magnetic information storage --Outlook based on analysis of magnetic recording mechanisms--, IEICE Trans. Elec., vol. E78-C, pp.1477-1492, 1995 